\title{
A 3D Non-stationary MmWave Channel Model for Vacuum Tube Ultra-High-Speed Train Channels
}

\author{
Yingjie $\mathrm{Xu}^{1,2}$, Kai $\mathrm{Yu}^{3}, \mathrm{Li} \mathrm{Li}^{4}$, Xianfu Lei ${ }^{4,1}$, Li Hao ${ }^{4}$, Cheng-Xiang Wang ${ }^{1,2, *}$ \\ ${ }^{1}$ National Mobile Communications Research Laboratory, School of Information of Science and Engineering, \\ Southeast University, Nanjing 210096, China. \\ ${ }^{2}$ Purple Mountain Laboratories, Nanjing 211111, China. \\ ${ }^{3}$ China Railway Eryuan Engineering Group Co. Ltd, Chengdu, Sichuan 610031, China. \\ ${ }^{4}$ School of Information Science and Technology, Southwest Jiao Tong University, Chengdu 610031, China. \\ *Corresponding Author: Cheng-Xiang Wang \\ Email: yjxu@seu.edu.cn, ekyukai@qq.com, \\ $\{115 \mathrm{e} 08$, xflei, lhao $\} @$ home.swjtu.edu.cn, chxwang@seu.edu.cn
}

\begin{abstract}
As a potential development direction of future transportation, the vacuum tube ultra-high-speed train (UHST) wireless communication systems have newly different channel characteristics from existing high-speed train (HST) scenarios. In this paper, a three-dimensional non-stationary millimeter wave (mmWave) geometry-based stochastic model (GBSM) is proposed to investigate the channel characteristics of UHST channels in vacuum tube scenarios, taking into account the waveguide effect and the impact of tube wall roughness on channel. Then, based on the proposed model, some important time-variant channel statistical properties are studied and compared with those in existing HST and tunnel channels. The results obtained show that the multipath effect in vacuum tube scenarios will be more obvious than tunnel scenarios but less than existing HST scenarios, which will provide some insights for future research on vacuum tube UHST wireless communications.
\end{abstract}

Index Terms-vacuum tube UHST channels, mmWave, GBSM, waveguide effect, non-stationarity

\section{INTRODUCTION}

With the social development and population growth, transportation is developing rapidly. In the future, vacuum tube transportation systems can overcome the limitations of current wheel-rail transportation environment such as air resistance and train wheel-rail resistance on the speed of trains, and the train speed can reach thousands of kilometers per hour [1], becoming an important development direction of HST transportation systems. For vacuum tube UHST train-toground wireless communication systems, the applications of the fifth generation $(5 \mathrm{G})$ wireless communication networks, which only support up to $500 \mathrm{~km} / \mathrm{h}$ mobility [2], are not enough. Accordingly, this will promote research on ultra high mobility in the sixth generation $(6 \mathrm{G})$ wireless communication networks [3].

There are many channel models which can well reflect the wireless channel propagation characteristics in existing HST and tunnel scenarios [4]-[7]. In [8] a deterministic channel model for HST scenarios was proposed, and channel small-scale fading characteristics were investigated. A mmWave massive MIMO GBSM for HST communication systems was shown in [9], where the author studied HST channel statistical properties. The channel statistical properties in tunnel scenarios were investigated in [10] by proposing a three-dimensional (3D) non-stationary GBSM. However, these models cannot be directly used for vacuum tube UHST channels. The vacuum and narrow space environment have newly different effects on its wireless channel, and the ultra high speed will bring larger Doppler frequency and more fast handovers [11]. In [12], a propagation graph channel model for vacuum tube UHST scenarios was proposed, and several channel properties were analyzed, such as multipath, $\mathrm{K}$ factor, and channel capacity. However, it focused on channels without considering mmWave technologies that can provide high data rate transmissions to communication systems. The general 3D non-stationary $5 \mathrm{G}$ wireless channel model in [13] can reflect channel characteristics of most scenarios. However, due to the assumption of random cluster distribution and missing consideration of the waveguide effect in channels, it cannot be directly applied to the vacuum tube UHST channel.

To the best of author's knowledge, non-stationary mmWave vacuum tube UHST channel models are still missing in the literature. To fill the research gaps, a 3D non-stationary mmWave GBSM for vacuum tube UHST scenarios is proposed in this paper. In the model, it is assumed that the scattering clusters are distributed on the inner wall of the tube, and then the vacuum tube UHST channel properties are studied.

The remainder of this paper is organized as follows. A 3D non-stationary mmWave GBSM is proposed in Section II and channel statistical properties are derived in Section III. Section IV illustrates simulation results and discussions about UHST channels. Finally, conclusions are shown in Section V.

\section{A 3D NON-STATIONARY MMWAVE MIMO GBSM FOR VACUUM TUbE UHST SCENARIOS}

A. Description of Vacuum Tube UHST Communication Network Architecture

The communication network architecture for vacuum tube UHST scenarios is shown in Fig. 1 In communication sys- 
Distributed Antenna System

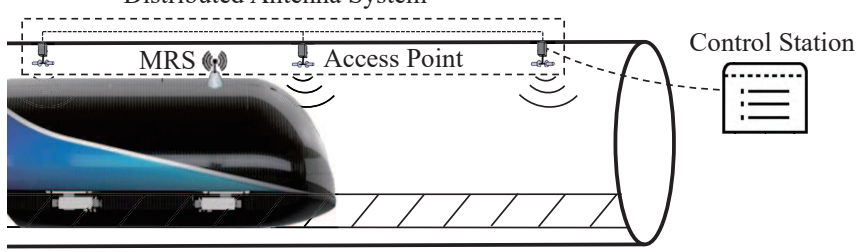

Fig. 1. A UHST network architecture for vacuum tube scenarios.

tems, it is considered to adopt technologies, such as distributed antenna system (DAS), radio over fiber (RoF), and mobile relay station (MRS) [10]. The access points (APs) are used and fixed on the top of the metal tube inner wall to form the DAS. They are connected by the RoF, and finally connected to the control station (CS) at the station. A small MRS is installed on surface of the train to reduce high penetration loss of the signal entering train compartment. By distributing the DAS and the MRS in communication systems, the propagation space between base stations and the train is divided into multiple parts. This paper will aim to investigate the channel between AP and MRS.

\section{B. The mmWave MIMO GBSM for Vacuum Tube UHST Sce- narios}

Let us consider a MIMO system with $Q$ and $P$ antenna elements at the receiver $(\mathrm{Rx})$ and transmitter (Tx) side and let $\mathbf{A}_{p}^{T}(t)$ and $\mathbf{A}_{q}^{R}(t)$ denote $3 \mathrm{D}$ position vectors of the $p$ th transmit antenna and the $q$ th receive antenna. Based on the 5G general channel model [13], a 3D non-stationray GBSM is proposed, where the channel propagation environment is characterized as a 3D cylindrical model with radius $R$. The Cartesian coordinate system is used to describe the location of the Tx and the Rx. It is assumed that the Rx (MRS) on train moves towards the Tx (AP), as shown in Fig. 2.

1) Channel Impulse Response: The complete channel matrix is given by $\mathbf{H}=[P L \cdot S H \cdot B L \cdot O L]^{\frac{1}{2}} \cdot \mathbf{H}_{s}$, where $P L$ represents the path loss, $S H$ represents the shadowing, $B L$ represents the blockage loss, and $O L$ represents the oxygen and molecular absorption loss. Widely used path loss model and shadowing model are shown in [14]. The blockage loss is caused by train and obstacles in vacuum tube UHST scenarios and its model is taken from [15] here. The oxygen and molecular absorption loss model for mmWave can be found in [16]. Note that the parameter values in above models should be measured additionally and will be different from those measured in the standard atmosphere because of the vacuum environment in UHST channels.

The small-scale fading can be denoted as a complex matrix $\mathbf{H}_{s}=\left[h_{p q}(t, \tau)\right]_{P \times Q}$, where $h_{p q}(t, \tau)$ is the channel impulse response (CIR) between $\mathbf{A}_{p}^{T}(t)$ and $\mathbf{A}_{q}^{R}(t)$ that consists of line-of-sight (LoS) component and non-line-of-sight (NLoS) components. The NLoS components include single-bounced (SB) and multi-bounced (MB) components. The propagation environment between $\mathrm{Tx}$ and $\mathrm{Rx}$ is abstracted by effective clusters that characterize the first and last bounce of the

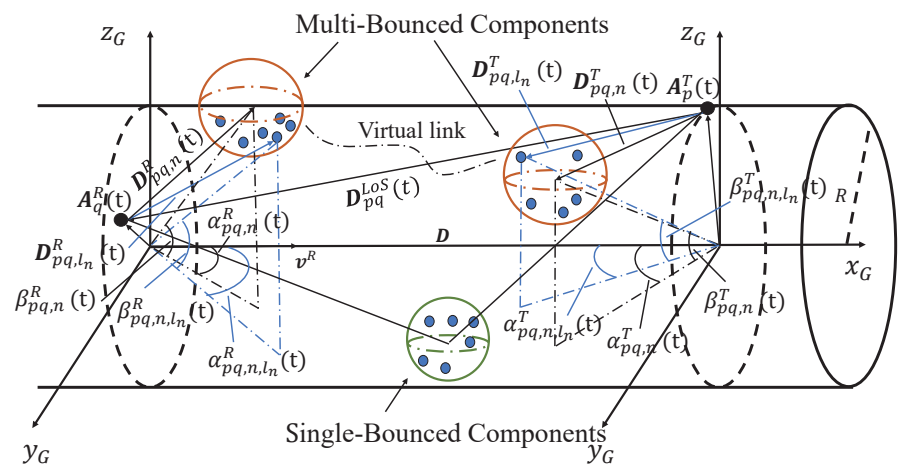

Fig. 2. A 3D non-stationary mmWave MIMO GBSM for vacuum tube UHST scenarios.

channel. Assuming there are a total of $N(t)$ effective clusters on tube wall at time $t$ in channel, and $L_{n}$ is the timevariant number of rays within Cluster $_{n}$. Note that each ray in each cluster should have its own power and delay to support higher spectral resolution in models, which is the difference between mmWave channels and conventional channels. The CIR $h_{p q}(t, \tau)$ can be expressed as [13]

$$
\begin{aligned}
h_{p q}(t, \tau) & =\underbrace{h_{p q}^{L o S}(t) \cdot \delta\left(\tau-\tau^{L o S}(t)\right)}_{L o S} \\
& +\underbrace{\sum_{n=1}^{N} \sum_{l_{n}=1}^{L_{n}} h_{p q, n, l_{n}}^{N L o S}(t) \cdot \delta\left(\tau-\tau_{n}(t)-\tau_{l_{n}}(t)\right)}_{N L o S} .
\end{aligned}
$$

In (1), $\tau^{\operatorname{LoS}}(t)$ is the delay of LoS component, $\tau_{n}(t)$ is the delay of Cluster ${ }_{n}$ and the delay $\tau_{l_{n}}(t)$ of the $l_{n}$ th rays within Cluster $_{n}$ is taken into account to support mmWave scenarios. Moreover, to model high mobility and non-stationarity of UHST channels, all parameters in the model are time-variant. Suppose the train speed vector at the $\mathrm{Rx}$ is $\mathbf{v}^{R}$, and initial distance vector between the Rx and Tx is D. The key channel parameters are defined in Table I.

For the LoS component, the complex channel $h_{p q}^{L o S}(t)$ can be written as

$$
h_{p q}^{L o S}(t)=\sqrt{\frac{K_{p q}(t)}{K_{p q}(t)+1}} e^{-\frac{j 2 \pi D_{p q}^{L o S}}{\lambda}} e^{j 2 \pi f_{p q}^{L o S}(t) \cdot t}
$$

where $f_{p q}^{L o S}(t)$ can be calculated as

$$
f_{p q}^{L o S}(t)=\frac{1}{\lambda} \frac{\left\langle\mathbf{D}_{p q}^{L o S}(t), \mathbf{v}^{R}\right\rangle}{\left\|\mathbf{D}_{p q}^{L o S}(t)\right\|} .
$$

The delay $\tau^{L o S}(t)$ can be expressed as

$$
\tau^{L o S}(t)=\frac{\left\|\mathbf{D}_{p q}^{L o S}(t)\right\|}{c}
$$

where $\|\cdot\|$ calculates the Frobenius norm and $\mathbf{D}_{p q, n, l_{n}}^{N L o S}(t)$ can be calculated as

$$
\mathbf{D}_{p q}^{L o S}(t)=\mathbf{A}_{q}^{R}(t)-\mathbf{A}_{p}^{T}(t) .
$$


TABLE I

DEFINITION OF Key PARAMETERS.

\begin{tabular}{|l|l|}
\hline Parameters & Definition \\
\hline $\mathbf{D}_{p q}^{L o S}(t)$ & 3D distance vector between $\mathbf{A}_{p}^{T}(t)$ and $\mathbf{A}_{q}^{R}(t)$ of the LoS component \\
\hline $\mathbf{D}_{p q}^{N L O S}(t)$ & 3D distance vector between $\mathbf{A}_{p}^{T}(t)$ and $\mathbf{A}_{q}^{R}(t)$ of the NLoS components \\
\hline $\mathbf{D}_{q p, n}^{R}(t), \mathbf{D}_{q p, n}^{T}(t)$ & 3D distance vectors between Cluster ${ }_{n}$ and the Rx (Tx) array center \\
\hline $\mathbf{D}_{p q, n, l_{n}}^{T}(t), \mathbf{D}_{p q, n, l_{n}}^{T}(t)$ & 3D distance vectors between the $l_{n}$ th rays within Cluster ${ }_{n}$ and the Rx (Tx) array center \\
\hline $\mathbf{D}$ & initial distance vector between Tx and Rx \\
\hline$\alpha_{p q, n}^{R}(t), \beta_{p q, n}^{R}(t)$ & azimuth and elevation angles between Cluster ${ }_{n}$ and the Rx array center \\
\hline$\alpha_{p q, n}^{T}(t), \beta_{p q, n}^{T}(t)$ & azimuth and elevation angles between Cluster ${ }_{n}$ and the Tx array center \\
\hline$\alpha_{p q, n, l_{n}}^{R}(t), \beta_{p q, n, l_{n}}^{R}(t)$ & azimuth and elevation angles between the $l_{n}$ th rays within Cluster $n$ and the Rx array center \\
\hline$\alpha_{p q, n, l_{n}}^{T}(t), \beta_{p q, n, l_{n}}^{T}(t)$ & azimuth and elevation angles between the $l_{n}$ th rays within Cluster $n$ and the Tx array center \\
\hline $\mathbf{A}_{q}^{R}(t), \mathbf{A}_{p}^{T}(t)$ & 3D position vectors of the $q$ th antenna at Rx and the $p$ th antenna at Tx \\
\hline $\mathbf{v}^{R}$ & 3D velocity vector of receive array \\
\hline$K_{p q}(t)$ & Rician factor \\
\hline$f_{p q}^{L O S}(t), f_{p q, n, l_{n}}^{N L O S}(t)$ & Doppler frequency between $\mathbf{A}_{p}^{T}(t)$ and $\mathbf{A}_{q}^{R}(t)$ of the LoS (NLoS) component \\
\hline$N(t)$ & the total number of effective clusters at time $t$ \\
\hline$P_{n, l_{n}}(t)$ & the normalized power of $l_{n}$ th rays within Cluster $n$ \\
\hline$\lambda$ & the wavelength of the signal \\
\hline
\end{tabular}

For NLoS components, the complex channel $h_{p q, n, l_{n}}^{N L o S}(t)$ can be written as

$$
\begin{aligned}
h_{p q, n, l_{n}}^{N L o S}(t) & =\sqrt{\frac{P_{n, l_{n}}(t)}{K_{p q}(t)+1}} e^{j\left(\varphi_{n, l_{n}}-\frac{2 \pi D_{p q, n, l_{n}}^{N L o S}(t)}{\lambda}\right)} \\
& \times e^{j 2 \pi f_{p q, n, l_{n}}^{N L o S}(t) \cdot t}
\end{aligned}
$$

where $\varphi_{n, l_{n}}$ is initial phase, $D_{p q, n, l_{n}}^{N \operatorname{LoS}}(t)$ and $f_{p q, n, l_{n}}^{N \operatorname{LoS}}(t)$ can be calculated as

$$
\begin{gathered}
D_{p q, n, l_{n}}^{N \operatorname{LoS}}(t)=\left\|\mathbf{D}_{p q, n, l_{n}}^{T}(t)\right\|+\left\|\mathbf{D}_{p q, n, l_{n}}^{R}(t)\right\|+\widetilde{\tau}_{n}(t) \cdot c \\
f_{p q, n, l_{n}}^{N L o S}(t)=\frac{1}{\lambda} \frac{\left\langle\mathbf{D}_{p q, n, l_{n}}^{R}(t), \mathbf{v}^{R}\right\rangle}{\left\|\mathbf{D}_{p q, n, l_{n}}^{R}(t)\right\|}
\end{gathered}
$$

where $\widetilde{\tau}_{n}(t) \cdot c$ represents the virtual link distance and $\widetilde{\tau}_{n}(t)$ is a virtual delay. The distance vectors $\mathbf{D}_{p q, n, l_{n}}^{R}(t)$ and $\mathbf{D}_{p q, n, l_{n}}^{T}(t)$ are determined by the AoAs and AoDs of $l_{n}$ th rays, as shown in (9) and (10).

$$
\begin{gathered}
\mathbf{D}_{p q, n, l_{n}}^{R}(t)=\frac{R}{\sqrt{1-\cos ^{2} \beta_{p q, n, l_{n}}^{R}(t) \cos ^{2} \alpha_{p q, n, l_{n}}^{R}(t)}} \\
{\left[\begin{array}{l}
\cos \beta_{p q, n, l_{n}}^{R}(t) \cos \alpha_{p q, n, l_{n}}^{R}(t) \\
\cos \beta_{p q, n, l_{n}}^{R}(t) \sin \alpha_{p q, n, l_{n}}^{R}(t) \\
\sin \alpha_{p q, n, l_{n}}^{R}(t)
\end{array}\right]^{T}+\mathbf{D}-\mathbf{A}_{q}^{R}(t)} \\
\mathbf{D}_{p q, n, l_{n}}^{T}(t)=\frac{R}{\sqrt{1-\cos ^{2} \beta_{p q, n, l_{n}}^{T}(t) \cos ^{2} \alpha_{p q, n, l_{n}}^{T}(t)}} \\
{\left[\begin{array}{l}
\cos \beta_{p q, n, l_{n}}^{T}(t) \cos \alpha_{p q, n, l_{n}}^{T}(t) \\
\cos \beta_{p q, n, l_{n}}^{T}(t) \sin \alpha_{p q, n, l_{n}}^{T}(t) \\
\sin \alpha_{p q, n, l_{n}}^{T}(t)
\end{array}\right]^{T}-\mathbf{A}_{q}^{T}(t) .}
\end{gathered}
$$

Similarly, the distance vectors $\mathbf{D}_{p q, n}^{R}(t)$ and $\mathbf{D}_{p q, n}^{T}(t)$ of Cluster $_{n}$ can be calculated by replacing $\alpha_{p q, n, l_{n}}^{R}(t)$, $\beta_{p q, n, l_{n}}^{R}(t)$ in (9) with $\alpha_{p q, n}^{R}(t), \beta_{p q, n}^{R}(t)$ and replacing $\alpha_{p q, n, l_{n}}^{T}(t), \beta_{p q, n, l_{n}}^{T}(t)$ in (10) with $\alpha_{p q, n}^{T}(t), \beta_{p q, n}^{T}(t)$.
2) Cluster Evolution in Time Domains for UHST channel: The cluster evolution in time domains for UHST channel is achieved by updating cluster information and geometric characteristics of channels. Here we use the birth and death process [9] to describe the time evolution of clusters, given appropriate cluster survival and recombination rates.

For survived clusters, firstly, which clusters are survived should be determined. Let $P_{T}(\Delta t)$ denote the survival probability of a cluster after $\Delta t$ and its calculation are given in [13]. Then, the position vector of receiving antenna is updated as

$$
\mathbf{A}_{q}^{R}(t+\Delta t)=\mathbf{A}_{q}^{R}(t)+\mathbf{v}^{R} \Delta t
$$

Similarly, the distance vectors in (5), (9) (12) need to be updated accordingly. Next, the delay of $\mathrm{Cluster}_{n}$ is updated as

$$
\begin{aligned}
& \tau_{n}(t+\Delta t) \\
& =\frac{\left\|\mathbf{D}_{p q, n, l_{n}}^{R}(t+\Delta t)\right\|+\left\|\mathbf{D}_{p q, n, l_{n}}^{T}(t+\Delta t)\right\|}{c}+\widetilde{\tau}_{m}(t+\Delta t)
\end{aligned}
$$

where the virtual delay $\widetilde{\tau}_{m}(t+\Delta t)$ at time $t+\Delta t$ is calculated as $\widetilde{\tau}_{n}(t+\Delta t)=e^{-\frac{\Delta t}{\varsigma}} \widetilde{\tau}_{n}(t)+\left(1-e^{-\frac{\Delta t}{\varsigma}}\right) X$. The random variable $X$ and $\widetilde{\tau}_{n}$ have the same distribution but are independent of each other and $\varsigma$ is a scenario-dependent parameter [13]. Finally, the mean power of $l_{n}$ th rays within Cluster $_{n}$ need to be updated as [10]

$$
\widetilde{P}_{n, l_{n}}(t+\Delta t)=\widetilde{P}_{n, l_{n}}(t) \frac{3 \tau_{n}(t)-2 \tau_{n}(t+\Delta t)+\tau_{l_{n}}}{\tau_{n}(t)+\tau_{l_{n}}} .
$$

It should be noted that the updated power in (13) should be normalized before being substituted into (6).

For new cluster generations, the number of new clusters generated at a stationary interval should be determined at first. In the fully enclosed tube, the channel keyhole effect [17] related to waveguide will cause the multipath components in signal to change with distance. As the distance between 
the $\mathrm{Rx}$ and $\mathrm{Tx}$ increases, the multipath components will experience more similar channel fadings. Moreover, it has been confirmed that the scattering surface roughness $\sigma_{h}$ will affect the scattering and reflection loss of the signal, and thus the number of multipaths reaching the Rx. By considering above phenomenons in vacuum tube channel, it is assumed that the number of new clusters generated at time $t$ follows a Poisson distribution, and its mean value is

$$
E\left[N_{\text {new }}(t)\right]=\frac{\lambda_{G}}{\lambda_{R}}\left(1-P_{T}(t)\right)\left(1-\frac{\left\|\mathbf{D}^{L o S}(t)\right\|}{D}\right) \frac{\rho_{s}}{\rho_{s 0}}
$$

where $\rho_{s 0}$ is the scattering coefficient when the roughness $\sigma_{h}=0$. The scattering coefficient $\rho_{s}$ is calculated as [18]

$$
\rho_{s}=e^{\left(-8\left(\frac{\pi \sigma_{h} \cos (\bar{\beta})}{\lambda}\right)^{2}\right)}
$$

where $\bar{\beta}$ is the mean elevation angle of the incident ray. After the number of new cluster determined, key parameters for new cluster need to be given. Here, the calculation of the ray power $P_{n, l_{n}}$ is referenced in [18]. The number of rays in cluster follows the Poisson distribution and the angle parameters follow the Von Mises distribution [10]. The virtual delay $\tau_{n}$ follows the exponential distribution [19].

\section{Statistical Properties}

In this section, several typical statistical properties of the mmWave channel model for vacuum tube UHST scenarios will be derived.

\section{A. The Time-Variant Transfer Function}

The time-variant transfer function $H_{p q}(t, f)$ is the Fourier transform of the CIR $h_{p q}(t, \tau)$ relative to $\tau$, which can be expressed as

$$
\begin{aligned}
H_{p q}(t, f) & =\int_{-\infty}^{\infty} h_{p q}(t, \tau) e^{-j 2 \pi \tau f} d \tau \\
& =h_{p q}^{\operatorname{LoS}}(t) e^{-j 2 \pi \tau_{L o S}(t) f} \\
& +\sum_{n=1}^{N} \sum_{l_{n}=1}^{L_{n}} h_{p q, l_{n}}^{N L o S}(t) e^{-j 2 \pi\left(\tau_{n}(t)+\tau_{l_{n}}(t)\right) f} .
\end{aligned}
$$

\section{B. Space-Time-Frequency Correlation Function}

In order to investigate the correlation of UHST channels, the space-time-frequency correlation function (STFCF) is calculated as

$$
\begin{aligned}
& R_{p q, p^{\prime} q^{\prime}}\left(\delta_{p}, \delta_{q}, \Delta f, \Delta t ; t, f\right) \\
& =E\left[H_{p q}(t, f) H_{p^{\prime} q^{\prime}}^{*}(t+\Delta t, f+\Delta f)\right]
\end{aligned}
$$

where $\delta_{p}=\left\|\mathbf{A}_{p}^{T}-\mathbf{A}_{p^{\prime}}^{T}\right\|, \delta_{q}=\left\|\mathbf{A}_{q}^{R}-\mathbf{A}_{q^{\prime}}^{R}\right\|$. Due to the LoS component is determined based on Tx and Rx's relative position while NLoS components are determined based on parameters which are randomly generated, for simplicity, it is assumed here that the LoS and NLoS components are uncorrelated [13]. Then (17) can be written as

$$
\begin{aligned}
& R_{p q, p^{\prime} q^{\prime}}\left(\delta_{p}, \delta_{q}, \Delta f, \Delta t ; t, f\right) \\
& =R_{p q, p^{\prime} q^{\prime}}^{L o S}\left(\delta_{p}, \delta_{q}, \Delta f, \Delta t ; t, f\right)+R_{p q, p^{\prime} q^{\prime}}^{N L o S}\left(\delta_{p}, \delta_{q}, \Delta f, \Delta t ; t, f\right) .
\end{aligned}
$$

The correlation function of the LoS component is expressed as follows [10]:

$$
\begin{aligned}
& R_{p q, p^{\prime} q^{\prime}}^{L o S}\left(\delta_{p}, \delta_{q}, \Delta f, \Delta t ; t, f\right) \\
& =\frac{K}{K+1} H_{p q}^{L o S}(t, f) \cdot H_{p^{\prime} q^{\prime}}^{L o S *}(t+\Delta t, f+\Delta f) .
\end{aligned}
$$

For NLoS components, the correlation function is expressed as

$$
\begin{aligned}
& R_{p q, p^{\prime} q^{\prime}}^{N o S}\left(\delta_{p}, \delta_{q}, \Delta f, \Delta t ; t, f\right) \\
& =\frac{1}{K+1} \sum_{n=1}^{N} \sum_{l_{n}=1}^{L_{n}} H_{p q}^{N L o S}(t, f) \cdot H_{p^{\prime} q^{\prime}}^{N L o S *}(t+\Delta t, f+\Delta f) .
\end{aligned}
$$

In STFCF, let $\Delta f=0, q=q^{\prime}, p=p^{\prime}$, the function will be reduced to the time-variant ACF. Let $\Delta t=0, \Delta f=0$, $q=q^{\prime}$ (or $p=p^{\prime}$ ), the function will be reduced to the timevariant cross-correlation function $(\mathrm{CCF}$ ) of the $\mathrm{Rx}$ (or Tx). Let $\Delta t=0, q=q^{\prime}, p=p^{\prime}$, the function will be reduced to the time-variant frequency correlation function (FCF).

\section{Stationary Interval}

The stationary interval is the minimum time interval during which the channel response remains constant. It can be used to determine the channel estimation frequency in ultra-highspeed mobile scenes [10]. It is defined as the maximum length of time that the ACF of the power delay profile (PDP) exceeds a certain threshold $\varsigma$, namely,

$$
\mathrm{I}=\inf \left\{\Delta t \mid R_{\Lambda}(t, \Delta t) \leq \varsigma\right\}
$$

where $\inf \{\cdot\}$ is the infimum of a function, $R_{\Lambda}(t, \Delta t)$ is the the ACF of the PDP and its calculation is given in [13]. The threshold $\varsigma$ can be adjusted according to certain scenario and set to $80 \%$ here.

\section{RESults And Discussions}

In this section, channel properties of the proposed channel model are simulated and analyzed. According to the size of existing vacuum tube train design of Hyperloop One [20], the cross section radius of vacuum tube is set $R=2 \mathrm{~m}$ here. The material of tube wall is low carbon steel [20] which can be approximated as a smooth surface, therefore the roughness is set $\sigma_{h}=0$ here. In metal tube, the position coordinates of the Tx is set as $\left(x_{T}, y_{T}, z_{T}\right)=(0,0,4)$ while the initial position coordinates of the $\mathrm{Rx}$ is set as $\left(x_{R}, y_{R}, z_{R}\right)=\left(D_{0}, 0,3\right)$, where $D_{0}$ is the initial distance. At the $\mathrm{Rx}$ and Tx, a $2 \times 2$ MIMO linear antenna array communication systems are taken into consideration and antenna spacing are set as $\Delta x_{T}=$ $\Delta x_{R}=\lambda$ [10]. Also, other parameters like carrier frequency $f_{c}=58 \mathrm{GHz}$ and the train speed $v^{R}=1080 \mathrm{~km} / \mathrm{h}$. In the calculation of $P_{T}(\Delta t)$, the generation and recombination rate are set as $r_{b}=80 / \mathrm{m}, r_{d}=4 / \mathrm{m}$, respectively. The remaining parameters are randomly generated with reference to the $5 \mathrm{G}$ general channel model [13], and the equal area method (EAM) [10] is used to obtain discrete AoA and AoD angle characteristics in channel model. 


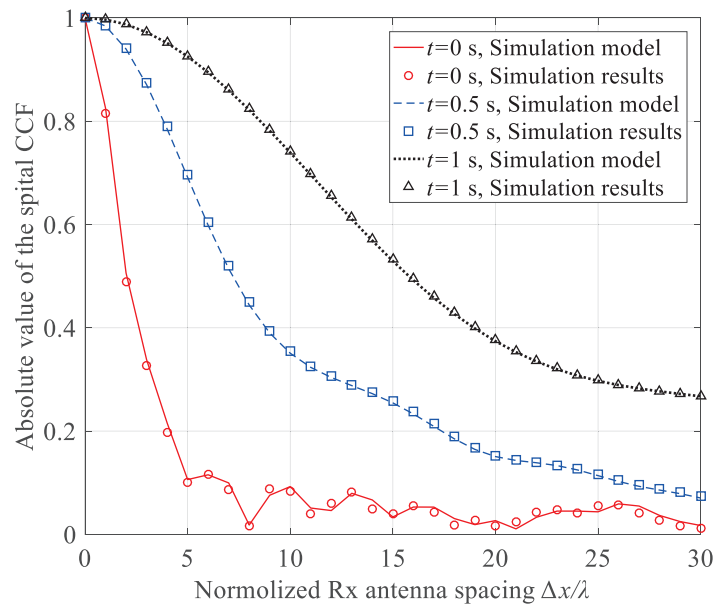

Fig. 3. Spatial CCFs comparison of simulation model and simulation results at different time instants $\left(R=2 \mathrm{~m}, \sigma_{h}=0, r_{b}=80 / \mathrm{m}, r_{d}=4 / \mathrm{m}\right.$, $\left.D_{0}=600 \mathrm{~m}, v^{R}=1080 \mathrm{~km} / \mathrm{h}, f_{c}=58 \mathrm{GHz}, k_{1}=k_{2}=6\right)$.

\section{A. The Time-Variant Spatial CCF}

The spatial CCFs comparison of the simulation model and simulation results at different time instants are shown in Fig. 3 . Since the parameters are time-variant, such as azimuth AoA and elevation AoA, the spatial cross-correlation characteristics are different at different time instants, and also, the simulation model and simulation results curve fit well.

\section{B. The Time-Variant ACF}

The ACFs comparison of simulation model and simulation results at different time instants are illustrated in Fig. 4 The curve fit of the simulation model and the simulation result is very good. The comparisons of ACFs of the simulation model for different $v^{R}$ at $t=0 \mathrm{~s}$ are shown in Fig. 5. As train speed increases, the ACF downward trend accelerates, and the attenuation is more rapid. In the future UHST scenarios, trains can reach thousands of kilometers per hour, which means the smaller coherence time will be considered in UHST channels.

\section{Comparison with Existing HST and Tunnel Channels}

In tunnel scenarios, the material of tunnel wall is generally reinforced concrete. Here, the roughness is set $\sigma_{h}=0.002$ [21] to simulate tunnel environment. The HST channel model in [9] is used to modeling existing HST channel here. Some channel characteristics compared in above scenarios are shown as follows.

The number of clusters changed with distance in three channels are illustrated in Fig. 6, Due to extremely small space environment of the vacuum tube and tunnel, the number of clusters in their channels is much less than that in HST channels, and the same phenomenon in tunnel wireless communication can be found in [12]. Moreover, compared with nearly smooth surface of vacuum tube, the signal will experience greater loss after passing through the rougher tunnel wall, which will reduce the effective propagation path

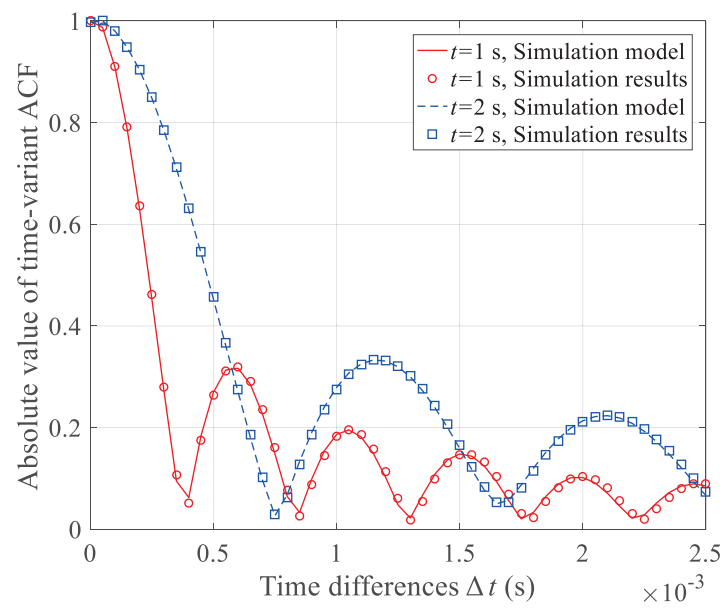

Fig. 4. ACFs comparison of simulation model and simulation results at different time instants $\left(R=2 \mathrm{~m}, \sigma_{h}=0, r_{b}=80 / \mathrm{m}, r_{d}=4 / \mathrm{m}\right.$, $\left.D_{0}=600 \mathrm{~m}, v^{R}=1080 \mathrm{~km} / \mathrm{h}, f_{c}=58 \mathrm{GHz}, k_{1}=k_{2}=6\right)$.

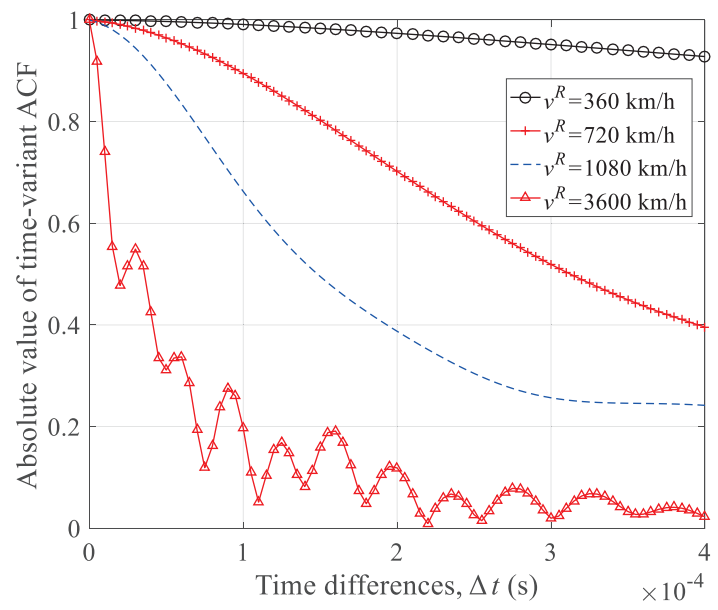

Fig. 5. Comparisons of ACFs of the simulation model for different $v^{R}$ at $t=0 \mathrm{~s}\left(R=2 \mathrm{~m}, \sigma_{h}=0, r_{b}=80 / \mathrm{m}, r_{d}=4 / \mathrm{m}, D_{0}=600 \mathrm{~m}, f_{c}=58\right.$ $\left.\mathrm{GHz}, k_{1}=k_{2}=6\right)$.

to the Rx [11]. Fig. 7 compares the stationary intervals of vacuum tube UHST scenarios with existing HST scenarios. In existing HST scenarios, at a train speed of $360 \mathrm{~km} / \mathrm{h}$ in 58 $\mathrm{GHz}$, the stationary interval is about $0.35 \mathrm{~ms}$, while it should be consider smaller in UHST channel, which is about 0.05 ms.

\section{COnClusions}

In this paper, a 3D non-stationary mmWave channel model for vacuum tube UHST communication systems has been proposed and its channel statistical properties have been studied, including time-variant ACF and spatial CCF. The results show that the non-stationarity of UHST channels. The simulation results match the simulation model well. Moreover, by comparing channel properties of vacuum tube UHST scenarios with existing tunnel and HST scenarios, it is found 


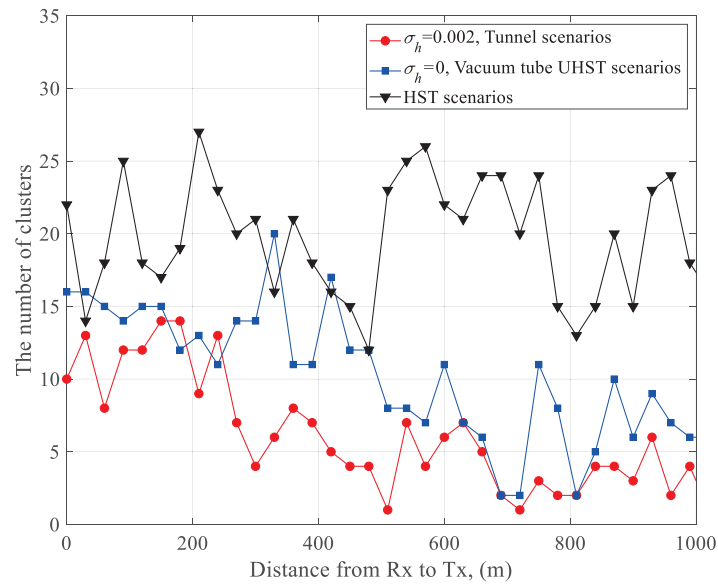

Fig. 6. Comparisons of the number of clusters in different scenarios $\left(D_{0}=\right.$ $\left.1000 \mathrm{~m}, v^{R}=1080 \mathrm{~km} / \mathrm{h}, f_{c}=58 \mathrm{GHz}, k_{1}=k_{2}=6\right)$.

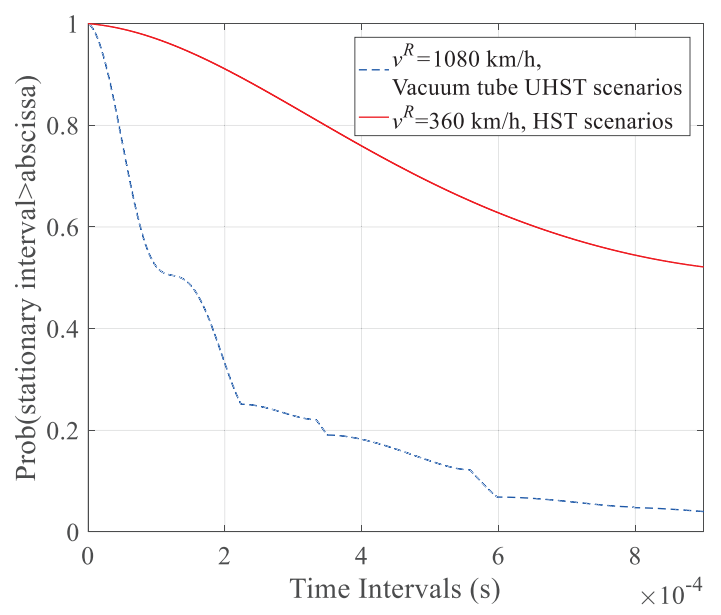

Fig. 7. Comparisons of empirical CCDFs of stationary intervals in different scenarios $\left(D_{0}=600 \mathrm{~m}, f_{c}=58 \mathrm{GHz}, k_{1}=k_{2}=6\right)$.

that there are more multipaths in vacuum tube UHST channel than tunnel channels but less than HST channels. For future work, more statistical properties of UHST channel need to be investigated and the available measured date also need to be considered to modify the model once there are some channel measurements on vacuum tube UHST scenarios.

\section{ACKNOWLEDGMENT}

This work was supported by the National Key R\&D Program of China under Grant 2018YFB1801101, the China Railway Eryuan Engineering Group Co. Ltd Project under Grant KYY2019110(19-21), the National Natural Science Foundation of China (NSFC) under Grant 61960206006, the Frontiers Science Center for Mobile Information Communication and Security, the High Level Innovation and Entrepreneurial Research Team Program in Jiangsu, the High Level Innovation and Entrepreneurial Talent Introduction Program in Jiangsu, the Research Fund of National Mobile Communications Research Laboratory, Southeast University, under Grant 2020B01, the Fundamental Research Funds for the Central Universities under Grant 2242020R30001, the EU H2020 RISE TESTBED2 project under Grant 872172, and the Open Research Fund of National Mobile Communications Research Laboratory, Southeast University under Grant 2021D05.

\section{REFERENCES}

[1] M. Jin and L. Huang, "Development status and trend of ultra high-speed vacuum pipeline transportation technology," Science and Technology China, vol. 5, no. 11, pp. 1-3, Mar. 2017.

[2] X.-H. You, C.-X. Wang, J. Huang, et al., "Towards 6G wireless communication networks: vision, enabling technologies, and new paradigm shifts," Sci. China Inf. Sci., vol. 41, no. 1, Jan. 2021.

[3] C.-X. Wang, J. Huang, H. Wang, X. Gao, X.-H. You, and Y. Hao, "6G wireless channel measurements and models: Trends and challenges," IEEE Veh. Technol. Mag., vol. 15, no. 4, pp. 22-32, Dec. 2020.

[4] C.-X. Wang, J. Bian, J. Sun, W. Zhang, and M. Zhang, "A survey of 5G channel measurements and models," IEEE Commun. Surveys Tuts., vol. 20, no. 4, pp. 3142-3168, 4th Quart., 2018.

[5] Y. Liu, C.-X. Wang, and J. Huang, "Recent developments and future challenges in channel measurements and models for $5 \mathrm{G}$ and beyond high-speed train communication systems," IEEE Commun. Mag., vol. 57, no. 9, pp. 50-56, Sept. 2019.

[6] Y. Liu, A. Ghazal, C.-X. Wang, X. Ge, Y. Yang, and Y. Zhang, "Channel measurements and models for high-speed train wireless communication systems in tunnel scenarios: a survey," Sci. China Inf. Sci., vol. 60, no. 8, pp. 1-17, Oct. 2017.

[7] C.-X. Wang, A. Ghazal, B. Ai, Y. Liu, and P. Fan, "Channel measurements and models for high-speed train communication systems: A survey," IEEE Commun. Surveys Tuts., vol. 18, no. 2, pp. 974-987, Apr.-Jun. 2016.

[8] K. Guan, B. Ai, B. Peng, D. He, G. Li, J. Yang, Z. Zhong, and T. Kürner, "Towards realistic high-speed train channels at $5 \mathrm{G}$ millimeterwave band-part II: Case study for paradigm implementation," IEEE Trans. Veh. Technol., vol. 67, no. 10, pp. 9129-9144, Oct. 2018.

[9] Y. Liu, C.-X. Wang, J. Huang, J. Sun, and W. Zhang, "Novel 3-D nonstationary mmwave massive mimo channel models for $5 \mathrm{G}$ highspeed train wireless communications," IEEE Trans. Veh. Technol., vol. 68, no. 3, pp. 2077-2086, Mar. 2019.

[10] Y. Liu, C.-X. Wang, C. F. Lopez, G. Goussetis, Y. Yang, and G. K. Karagiannidis, "3D non-stationary wideband tunnel channel models for 5G high-speed train wireless communications," IEEE Trans. Intell. Transp. Syst., vol. 21, no. 1, pp. 259-272, Jan. 2020.

[11] C. Qiu, L. Liu, Y. Liu, Z. Li, J. Zhang, and T. Zhou, "Key technologies of broadband wireless communication for vacuum tube high-speed flying train," in Proc. IEEE VTC, Kuala Lumpur, Malaysia, Apr. 2019, pp. $1-5$.

[12] B. Han, J. Zhang, L. Liu, and C. Tao, "Position-based wireless channel characterization for the high-speed vactrains in vacuum tube scenarios using propagation graph modeling theory," Radio Science, vol. 55, no. 4, pp. 1-12, Apr. 2020.

[13] S. Wu, C.-X. Wang, e. M. Aggoune, M. M. Alwakeel, and X. You, "A general 3-D non-stationary 5G wireless channel model," IEEE Trans. Commun., vol. 66, no. 7, pp. 3065-3078, Jul. 2018.

[14] Aalto University et al., "5G channel model for bands up to $100 \mathrm{GHz}$," v2.0, Mar. 2014.

[15] V. Nurmela et al., METIS, ICT-317669/D1.4, "METIS Channel Models," Jul. 2015.

[16] ITU-R, "Preliminary draft new report ITU-R M. [IMT-2020.EVAL]," Niagara Falls, Canada, R15-WP5D-170613-TD-0332, Jun. 2017.

[17] L. Liu, C. Qiu, Z. Li, B. Han, Y. Liu, and T. Zhou, "Thoughts on key technologies of broadband wireless communication for high-speed vacuum pipeline flying train," J. The China Railway Soc., vol. 41, no. 1, pp. 65-73, Jan. 2019.

[18] P. Kyösti et al. (Sep. 2007). WINNER II Channel Models, Ver-sion 1.1. [Online]. Available: http://www.ist-winner.org/WINNER2-Deliverables/D1.1.2v1.1.pdf

[19] Y. Liu, C.-X. Wang, C. F. Lopez, and X. Ge, "3D non-stationary wideband circular tunnel channel models for high-speed train wireless communication systems," Sci. China Inf. Sci., vol. 60, no. 8, pp. 1-13, Aug. 2017.

[20] Z. Feng, X. Fang, H. Li, A. Cheng, and Y. Pan, "Technological development of high speed maglev system based on low vacuum pipeline," Engineering Science, vol. 20, no. 6, pp. 105-111, June 2019.

[21] H. Wei, G. Zheng, and M. Jia, "The measurements and simulations of millimeter wave propagation at $38 \mathrm{GHz}$ in circular subway tunnels," in Proc. IEEE CJMW, Shanghai, China, Sep. 2008, pp. 51-54. 